\title{
A Comprehensive Study of Three Delay Compensation Algorithms for Flight Simulators
}

\author{
Liwen Guo ${ }^{*}$ and Frank M. Cardullo ${ }^{\dagger}$ \\ State University of New York at Binghamton, Binghamton \\ Jacob A. Houck \\ NASA Langley Research Center, Hampton, VA \\ Lon C. Kelly ${ }^{\S}$ \\ Unisys Corp., Hampton, VA \\ and \\ Thomas E. Wolters ${ }^{* *}$ \\ NASA Langley Research Center, Hampton, VA
}

\begin{abstract}
This paper summarizes a comprehensive study of three predictors used for compensating the transport delay in a flight simulator; The McFarland, Adaptive and State Space Predictors. The paper presents proof that the stochastic approximation algorithm can achieve the best compensation among all four adaptive predictors, and intensively investigates the relationship between the state space predictor's compensation quality and its reference model. Piloted simulation tests show that the adaptive predictor and state space predictor can achieve better compensation of transport delay than the McFarland predictor.
\end{abstract}

\section{Nomenclature}

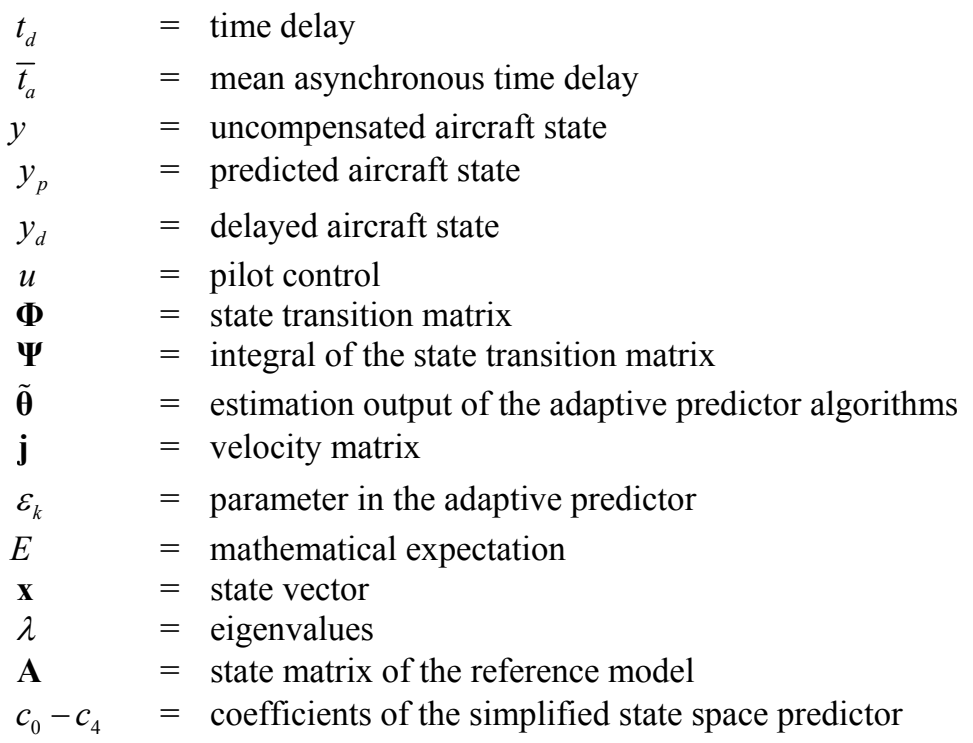

\footnotetext{
* Postdoctoral researcher, Department of Mechanical Engineering, AIAA Member

${ }^{\dagger}$ Professor, Department of Mechanical Engineering, Associate Fellow AIAA

*Branch Head, Associate Fellow AIAA

$\S$ Software Engineer, AIAA Member

${ }^{* *}$ Computer Engineer
} 


\section{Introduction}

T $\mathrm{N}$ the AIAA conference paper "New Predictive Filters for Compensating the Transport Delay on a Flight Simulator" published in 2004 (AIAA-5441 (2004) ${ }^{1}$, two novel predictors for compensating the transport delay in a flight simulator were introduced: the adaptive predictor and the state space predictor. The adaptive predictor makes use of the Kalman filter algorithm in a unique manner so that the predictor can provide the desired amount of prediction, significantly reducing the large spikes in the response, caused by the McFarland predictor. It also presents three simplified Kalman filter algorithms, i.e., the Karzmarz algorithm, the stochastic approximation algorithm, and the least mean squares algorithm. For the convenience of comparison with the McFarland compensator ${ }^{2}$, three consecutive steps of velocity are employed in the predictors. Therefore, the adaptive predictors and the McFarland predictor share the same structural expression;

$$
y_{p}(k)=y(k)+b_{0} v(k)+b_{1} v(k-1)+b_{2} v(k-2)
$$

where $y$ is the aircraft state to be predicted, $v$ is the corresponding velocity, $y_{p}$ is the predicted aircraft state, $k$ is the iteration index. Different means of determining the coefficients $b_{0}, b_{1}$ and $b_{2}$ define the predictor types. The McFarland predictor coefficients are designed with sinusoidal tuning, and remain constant throughout the whole simulation. In contrast to the McFarland predictor, the adaptive predictors update the coefficients during each frame. If $\tilde{\boldsymbol{\theta}}(k)=\left[\begin{array}{lll}b_{0}(k) & b_{1}(k) & b_{2}(k)\end{array}\right]^{T}$ and $\mathbf{j}^{T}(k)=\left[\begin{array}{lll}v(k) & v(k-1) & v(k-2)\end{array}\right]$, then all four adaptive predictors can be expressed in a general mathematical expression;

$$
\tilde{\boldsymbol{\theta}}(k)=\tilde{\boldsymbol{\theta}}(k-1)+\frac{\mathbf{j}(k)}{\varepsilon_{k}}\left[y(k)-y_{d}(k)-\mathbf{j}^{T}(k) \tilde{\boldsymbol{\theta}}(k-1)\right]
$$

Table 1. Expression of $\varepsilon_{k}$ in Eq. (2) for several where for different adaptive predictors, the only adaptive predictors

\begin{tabular}{|c|c|}
\hline Predictor & Expression of $\varepsilon_{k}$ \\
\hline Kalman Filter & $\sum_{i=k_{0}}^{k} \mathbf{j}(i) \mathbf{j}^{T}(i)$ \\
\hline $\begin{array}{c}\text { Kalman with forgetting } \\
\text { factor }\end{array}$ & $\sum_{i=k_{0}}^{k} \lambda^{i-k} \mathbf{j}(i) \mathbf{j}^{T}(i)$ \\
\hline Karzmarz & $\mathbf{j}^{T}(i) \mathbf{j}(i)$ \\
\hline Stochastic approximation & $\sum_{i=k_{0}}^{k} \mathbf{j}^{T}(i) \mathbf{j}(i)$ \\
\hline Least mean square & $\varepsilon$ \\
\hline
\end{tabular}
difference lies in the expression for $\varepsilon_{k}$. Table 1 lists the expressions for $\varepsilon_{k}$ for several adaptive predictors. Note the difference between the basic Kalman filter ( $2^{\text {nd }}$ row), in which $\varepsilon_{k}$ represents a square matrix, and the stochastic approximation algorithms $\left(5^{\text {th }}\right.$ row), which $\varepsilon_{k}$ represents a scalar. Notice also that this term becomes a constant for the least mean square algorithm.

With some examples, the paper AIAA-5441 (2004) shows that the adaptive predictors can significantly reduce the large spikes caused by the McFarland compensator, and the stochastic approximation algorithm achieves the best compensation among all adaptive algorithms. However, the paper did not investigate the underlying causes of the results. In the next section, this paper analyzes how the McFarland compensator produces large spikes, how the adaptive predictors mitigates these spikes, and finally presents a mathematical explanation for why the stochastic approximation algorithm works better than other adaptive predictors in compensating the transport delay by using its asymptotic ODE.

The second novel predictor is the state space predictor that is, loosely speaking, an application of the Sobiski/Cardullo ${ }^{3,4}$ compensator in a flight simulator using a linear, time-invariant aircraft model called a reference model. The Sobiski/Cardullo predictor is given by

$$
\mathbf{x}\left(t+t_{d}\right)=\mathbf{\Phi} \mathbf{x}(t)+\boldsymbol{\Psi B} u(t) .
$$

where $\boldsymbol{\Phi}=e^{\mathbf{A}_{d}}$ and $\boldsymbol{\Psi}=\int_{0}^{t_{d}} e^{\mathbf{A}\left(t_{d}-\tau\right)} d \tau$ are the state transition matrix and its integral matrix, where $\mathbf{A}$ is the state matrix of a linear system. A linear reference model is used to implement the Sobiski/Cardullo predictor when the 
system dynamics are not linear, and for such a situation, the matrix $\mathbf{A}$ is the state matrix of the reference model. Assume a $4^{\text {th }}$-order reference model has the following typical form:

$$
H_{A C}(s)=\frac{\beta_{2} s^{2}+\beta_{1} s+\beta_{0}}{s^{4}+\alpha_{3} s^{3}+\alpha_{2} s^{2}+\alpha_{1} s+\alpha_{0}}
$$

then the state space predictor can be simplified to ([1])

$$
y_{p}=\left(\phi_{11}+\phi_{12} \alpha_{3}+\phi_{13} \alpha_{2}\right) y+\left(\phi_{12}+\phi_{13} \alpha_{3}\right) \dot{y}+\phi_{13} \ddot{y}+\left(\psi_{1}-\phi_{13} \beta_{2}\right) u+\phi_{14} \beta_{0} \int_{0}^{T} u d t,
$$

in which $T$ is the frame length, and the $\phi_{i j}$ and $\psi_{j}$ are elements of matrices $\boldsymbol{\Phi}$ and $\boldsymbol{\Psi}$, which are functions of the $\alpha^{\prime} s \& \beta^{\prime} s$. Therefore, the coefficients of the five terms on the right side of Eq. (5) are closely related to the reference model. In other words, the quality of prediction of the state space predictor is determined by the choice of the reference model. This paper also investigates the relationship between the prediction quality of a state space compensator and the reference model it uses.

\section{Analyses of the Adaptive Predictor}

The biggest problem with the McFarland filter is the large spikes it produces. These spikes, stimulated by high frequency dynamics, are observable by the pilot in the visual scene. The longer the delay, the larger the magnitude of the spikes. The McFarland algorithm is a special integrator or an extrapolator. The simplest extrapolator to provide a prediction of $t_{d}$ is

$$
y_{p}(k)=y(k)+t_{d} v(k)
$$

If the velocity changes slowly (low frequency), this extrapolator works well, but if the velocity changes quickly, the prediction introduces error because the velocity may be quite different $t_{d}$ later. The McFarland filter is superior to the extrapolator given by Eq. (6) because it uses three consecutive steps of velocity that can extrapolate the future position better than a single velocity. Furthermore, the sinusoidal tuning of the coefficients contributes to the superiority of the McFarland compensator over others. For moderate frequencies (around $1 \mathrm{~Hz}$ ), let the average of these three velocities be $\bar{v}$, then Eq. (1) reduces

to $y_{p}(k)=y(k)+t_{d} \bar{v}(k)$, which is similar to Table 2. Several iterations of McFarland prediction with
Eq.(6), but the average velocity is used, resulting in spikes Eq.(6), but the average velocity is used, resulting in a better prediction. Therefore, its working frequency range is wider than the simplest extrapolator given in Eq. (6). However, spikes occur if the velocity changes rapidly (the frequency is higher). No matter what the delay is, the three coefficients $b_{0}, b_{1}$ and $b_{2}$ are always positive, negative and positive, respectively, and the absolute value of $b_{1}$ is always the largest, e.g., for $t_{d}=0.2 \mathrm{~s}$, $b_{0}=2.9979, b_{1}=-5.5197$ and $b_{2}=2.7219$. The absolute values of them are at least 10 times greater than $t_{d}$. If the velocity changes by more than $10 \%$ in several iterations, spikes in the predicted value are likely to occur. Table 2 gives an example, where the two spikes are highlighted in orange color.

\begin{tabular}{|c|c|c|c|c|}
\hline $\begin{array}{c}t \\
(\mathrm{~s})\end{array}$ & $\begin{array}{c}v_{1} \\
(\mathrm{rad} / \mathrm{s})\end{array}$ & $\begin{array}{c}v_{2} \\
(\mathrm{rad} / \mathrm{s})\end{array}$ & $\begin{array}{c}y_{p} \\
(\mathrm{rad} / \mathrm{s})\end{array}$ \\
\hline 59.776 & 0.1000 & 0.1015 & 0.1029 & 0.0115 \\
\hline 59.792 & 0.0877 & 0.1000 & 0.1015 & -0.02 \\
\hline 59.808 & 0.0754 & 0.0877 & 0.1000 & 0.0085 \\
\hline 59.824 & 0.0630 & 0.0754 & 0.0877 & 0.0072 \\
\hline 59.840 & 0.0507 & 0.0630 & 0.0754 & 0.0056 \\
\hline 59.856 & 0.0504 & 0.0507 & 0.0630 & 0.0402 \\
\hline 59.872 & 0.0502 & 0.0504 & 0.0507 & 0.0080 \\
\hline
\end{tabular}

From this example, the spikes in the McFarland compensation are caused by: first, the three coefficients change sign alternatively, and with alternative sign changes, the greater the sum $\sum_{i=0}^{2}\left|b_{i}\right|=\left|b_{0}\right|+\left|b_{1}\right|+\left|b_{2}\right|$ is, the more likely the 
equation to generate spikes; secondly, the coefficients do not change with respect to the simulation conditions. One better choice for the three coefficients would be $b_{0}=b_{1}=b_{2}=t_{d} / 3$. If, however, the coefficients are made to vary with the simulation conditions around $t_{d} / 3$, the prediction will be even better.

Although the adaptive predictors cannot guarantee non-alternative sign changes, they indeed significantly reduce the sum $\sum_{i=0}^{2}\left|b_{i}\right|=\left|b_{0}\right|+\left|b_{1}\right|+\left|b_{2}\right|$, and make the coefficients adaptable to the velocity changes. These are the two main reasons the adaptive predictors reduce the spikes, and cause smaller gain error than the McFarland predictor, especially for long time delay. While the adaptive predictors minimize the root mean squared gain error in every iteration, the coefficients are adjusted to some optimal pattern, so that even in the case of large velocity changes, such as the two cases shown in Table 2, spikes are less likely to occur.

Table 3. The three coefficients calculated with different

The fact that the adaptive predictors can $\operatorname{methods}\left(t_{d}=192 \mathrm{~ms}\right)$

significantly reduce the sum $\sum_{i=0}^{2}\left|b_{i}\right|=\left|b_{0}\right|+\left|b_{1}\right|+\left|b_{2}\right|$

is illustrated in Table 3, which was obtained by averaging the converged coefficients across 16 offline tests for all adaptive predictors. This sum is about 10 times larger for the McFarland predictor than for the basic Kalman predictor, which is still considerably greater than the stochastic approximation algorithms and the least mean algorithm. The basic Kalman filter and the Karzmarz algorithms still result in alternative coefficient sign changes. Although the least mean square algorithm gives all-positive coefficients in this table, but it has been demonstrated in other

\begin{tabular}{|c|c|c|c|c|c|}
\hline \hline Algorithm & $b_{0}$ & $b_{1}$ & $b_{2}$ & Sum & $\sum_{i=0}^{2}\left|b_{i}\right|$ \\
\hline $\begin{array}{c}\text { McFarland } \\
\text { Filter }\end{array}$ & 2.8613 & -5.2342 & 2.5650 & 0.192 & $\begin{array}{c}10.660 \\
5\end{array}$ \\
\hline $\begin{array}{c}\text { Kalman } \\
\text { Filter }\end{array}$ & -0.0030 & -0.5894 & 0.7811 & 0.189 & 1.3735 \\
\hline $\begin{array}{c}\text { Stochastic } \\
\text { Approximation }\end{array}$ & 0.0525 & 0.0571 & 0.0789 & 0.188 & 0.188 \\
\hline $\begin{array}{c}\text { Karzmarz } \\
\text { Algorithm }\end{array}$ & -0.2064 & 0.0503 & 0.3485 & 0.192 & 0.6052 \\
\hline $\begin{array}{c}\text { Least Mean } \\
\text { Square }\end{array}$ & 0.0284 & 0.0700 & 0.1066 & 0.205 & 0.205 \\
\hline \hline
\end{tabular}
simulations that the coefficients of the least mean square algorithm have alternative sign changes. Only the stochastic approximation algorithm guarantees non-alternative sign changes of the coefficients, and hence produces the smallest sum $\sum_{i=0}^{2}\left|b_{i}\right|$, and the coefficients are closer to $t_{d} / 3$ than other adaptive predictors. This may be shown mathematically as follows. For convenience, the expression of the stochastic approximation used in the final piloted tests is given in Eq. (7)

$$
\tilde{\boldsymbol{\theta}}(k)=\tilde{\boldsymbol{\theta}}(k-1)+\frac{\mathbf{j}(k)}{\sum_{i=k_{0}}^{k} \mathbf{j}^{T}(i) \mathbf{j}(i)}\left[y(k)-y_{d}(k)-\mathbf{j}^{T}(k) \tilde{\boldsymbol{\theta}}(k-1)\right]
$$

which was obtained by replacing $\varepsilon_{k}$ in Eq. (2) with $\sum_{i=k_{0}}^{k} \mathbf{j}^{T}(i) \mathbf{j}(i)$. This is just one version of a large family of stochastic approximation algorithms. Two other commonly used versions are the Saridis and Stein's algorithm and the Kwatny's form ${ }^{5}$. Robins and Monro have shown that the general Kalman filter algorithm given in Eq. (2) is a stochastic approximation algorithm if

$$
\varepsilon_{k}>0, \lim _{k \rightarrow \infty} \frac{1}{\varepsilon_{k}}=0, \sum_{k=1}^{\infty} \frac{1}{\varepsilon_{k}}=\infty \text {, and } \sum_{k=1}^{\infty} \frac{1}{\varepsilon_{k}^{2}}<\infty
$$

Eq. (8) also gives the conditions of algorithm convergence. Under normal circumstances, the velocity in a flight simulation does not change exponentially, and therefore the parameter $\varepsilon_{k}=\sum_{i=k_{0}}^{k} \mathbf{j}^{T}(i) \mathbf{j}(i)$ in Eq. (7) satisfies Eq. (8), and hence the algorithm of Eq. (7) is indeed a stochastic approximation algorithm. On the other hand, in the 
Karzmarz's algorithm $\varepsilon_{k}=\sum_{i=k_{0}}^{k} \mathbf{j}^{T}(i) \mathbf{j}(i)$, and in the least mean square algorithm $\varepsilon_{k}$ is a constant, both of which do not meet the $\lim _{k \rightarrow \infty} \frac{1}{\varepsilon_{k}}=0$ requirement. Therefore, they are not stochastic approximation algorithms.

The ODE that characterizes the asymptotic behavior of the stochastic approximation algorithm is ${ }^{6}$ given in Eq. (9)

$$
\begin{aligned}
& \dot{\boldsymbol{\theta}}=-\frac{1}{2} \frac{\partial}{\partial \boldsymbol{\theta}} E\left\{\left[y-y_{d}\right]-\mathbf{j}^{T} \boldsymbol{\theta}\right\}^{2} \\
& \dot{\boldsymbol{\theta}}=E\left(\mathbf{j} \mathbf{j}^{T}\right)-E\left(y \mathbf{j}^{T}\right) \boldsymbol{\theta}
\end{aligned}
$$

where " $E$ " is the mathematical expectation. The right side of the first equality of Eq. (9) is the negative gradient of the cost function, indicating that the stochastic approximation algorithm may be interpreted as a "stochastic" gradient descent algorithm. Though the gradient of the cost function $I=\frac{1}{2} E\left[y-y_{c}\right]^{2}\left(y_{c}\right.$ is the compensated $y$ ) with respect to $\boldsymbol{\theta}$ is unknown, the gradient at the current sample of $-\left\{\left[y(k)-y_{d}(k)\right]-\mathbf{j}^{T}(k) \boldsymbol{\theta}(k-1)\right\}^{2}$ is just $\mathbf{j}(k)\left[y(k)-y_{d}(k)-\mathbf{j}^{T}(k) \boldsymbol{\theta}(k-1)\right]$, the dynamic term in Eq. (7). Because the quantity inside the brackets $y(k)-y_{d}(k)-\mathbf{j}^{T}(k) \boldsymbol{\theta}(k-1)$ is a scalar, from the asymptotic ODE, $\dot{\boldsymbol{\theta}}(k)$ is in the direction of $\mathbf{j}(k)$. This means that the modifying term of $\boldsymbol{\theta}(k+1)$ is in the direction of $\mathbf{j}(k)$. And since the recursive algorithm starts with an initial zero vector, it is logical that $\boldsymbol{\theta}$ is in a direction close to the average of $\mathbf{j}$. Because $\mathbf{j}^{T}(k)=\left[\begin{array}{lll}v(k) & v(k-1) & v(k-2)\end{array}\right]$, the average velocity does not change much within two iterations, and the three elements of $\boldsymbol{\theta}$ (or the coefficients of the adaptive predictor) do not differ much. This demonstrates why the stochastic approximation algorithm gives the best prediction.

\section{Analyses of the State Space Predictor}

For all four $4^{\text {th }}$-order aircraft reference models that were applied to the state space compensator, the last two terms in Eq. (5) are very small compared with the first three terms, which come from the first term of Eq. (3). This shows that, in the state space compensation governed by Eq. (3), the second term contributes little to the prediction. In other words, the first term including the state transition matrix dominates the compensation. The state transition matrix plays a critical role in the predictive algorithm. By definition, the state transition matrix can be expressed as a Taylor series expansion

$$
\mathbf{\Phi}=e^{\mathbf{A} t_{d}}=\sum_{i=0}^{\infty} \frac{\left(\mathbf{A} t_{d}\right)^{i}}{i !}
$$

In this sense, the state space compensation may be viewed as a Taylor series extrapolation in the state space form.

Because this series is always convergent, it is usually approximated by truncating it to a finite series. The number of terms necessary to obtain satisfactory approximation depends on the matrix $\mathbf{A}$. For the four $4^{\text {th }}$-order aircraft reference models, the first five terms are sufficient. Therefore,

$$
\mathbf{\Phi}=e^{\mathbf{A} t_{d}} \approx \mathbf{I}+\mathbf{A} t_{d}+\frac{1}{2}\left(\mathbf{A} t_{d}\right)^{2}+\frac{1}{6}\left(\mathbf{A} t_{d}\right)^{3}+\frac{1}{24}\left(\mathbf{A} t_{d}\right)^{4}
$$

In fact, the fifth term in the last equation contributes little to the final result. This can be shown by using of the Cayley-Hamilton theorem. For a $4^{\text {th }}$-order matrix $\mathbf{A}$, the Cayley-Hamilton theorem states 


$$
\boldsymbol{\Phi}=e^{\mathbf{A} t_{d}}=\sum_{i=0}^{3} \alpha_{i} \mathbf{A}^{i}
$$

where the coefficients $\alpha_{0}-\alpha_{3}$ are the solution of the following coupled linear equations

$$
\sum_{i=0}^{3} \alpha_{i} \lambda_{j}^{i}=\exp \left(\lambda_{j} t_{d}\right),(j=0 \ldots 3)
$$

with $\lambda_{j}$ being the eigenvalues of the matrix $\mathbf{A}$, provided that all eigenvalues are distinct. For all four reference models, it has been found that

$$
\alpha_{0} \approx 1, \alpha_{1} \approx t_{d}, \alpha_{2} \approx \frac{1}{2} t_{d}^{2}, \alpha_{3} \approx \frac{1}{6} t_{d}^{3}
$$

This illustrates that Eqs. (10) and (11) are almost equivalent, with the only difference lying in the fifth term of Eq. (10). Using Eq. (10), the coefficients of the five terms in the right side of Eq. (5) were derived and are listed in Table 4. Investigation of all state space predictors shows that for correct prediction, the coefficient of the proportional term must be either unity or close to unity, the coefficient of the derivative term is equal to or very close to the time delay, and all other coefficients are much smaller. From Table 4, $a_{1}$ must be smaller than an upper limit that varies with the amount of time delay to be compensated, in order for the coefficient $c_{0}$ to be close to unity. Usually $a_{1}$ must be small, especially for a long time delay. For the same reason, $a_{2}$ also must be fairly small to make the coefficient $c_{0}$ close to the time delay $t_{d}$.

The restrictions on $a_{1}$ and $a_{2}$ indicate that there must also be some restrictions on the eigenvalues of the reference model. For a $4^{\text {th }}$-order model, the following relationships between the four eigenvalues $\lambda_{0}-\lambda_{3}$ and $a_{1}, a_{2}$ are held
Table 4. Approximate expressions of the coefficients of the simplified state space predictor with $\mathbf{a ~}^{\text {th }}$-order reference model

\begin{tabular}{|c|c|}
\hline Coefficient & Expression \\
\hline$c_{0}$ & $1-\frac{a_{1}}{6} t_{d}^{3}+\frac{a_{1} a_{3}-a_{0}}{24} t_{d}^{4}$ \\
\hline$c_{1}$ & $t_{d}-\frac{a_{2}}{6} t_{d}^{3}+\frac{a_{2} a_{3}-a_{1}}{24} t_{d}^{4}$ \\
\hline$c_{2}$ & $\frac{1}{2} t_{d}^{2}-\frac{a_{3}}{6} t_{d}^{3}+\frac{a_{3}^{2}-a_{2}}{24} t_{d}^{4}$ \\
\hline$c_{3}$ & $\left(\frac{a_{1}}{6} t_{d}^{3}-\frac{a_{3}}{24} t_{d}^{4}\right) b_{2}+\frac{b_{0}}{24} t_{d}^{4}$ \\
\hline$c_{4}$ & $\left(\frac{1}{6} t_{d}^{3}-\frac{a_{3}}{24} t_{d}^{4}\right) b_{0}$ \\
\hline
\end{tabular}

$$
\left\{\begin{array}{l}
\lambda_{0} \lambda_{1}+\lambda_{1} \lambda_{2}+\lambda_{2} \lambda_{3}+\lambda_{0} \lambda_{3}+\lambda_{1} \lambda_{3}+\lambda_{0} \lambda_{2}=a_{2} \\
\lambda_{0} \lambda_{1} \lambda_{2}+\lambda_{1} \lambda_{2} \lambda_{3}+\lambda_{0} \lambda_{1} \lambda_{3}+\lambda_{0} \lambda_{2} \lambda_{3}=-a_{1}
\end{array}\right.
$$

For a stable model, all eigenvalues must have negative real parts. Because all eigenvalues have the same sign, Eq. (14) means

$$
\left\{\begin{array}{l}
\left|\lambda_{i} \lambda_{j}\right|<a_{2},(i \neq j) \\
\left|\lambda_{i} \lambda_{j} \lambda_{k}\right|<a_{1},(i \neq j \neq k)
\end{array}\right.
$$

It shows the eigenvalues of the reference model must be smaller than some upper limits in order for the state space predictor to function properly. Because the eigenvalues of a model are closely related to its bandwidth, the bandwidth may also meet some conditions. This can be verified by gradually varying the bandwidth of a reference model and see how the state space compensation is affected. Fig. 1 shows the results. Investigation reveals that satisfactory compensation in simulating the Boeing 757 can be achieve when the bandwidth is below $1 \mathrm{rad} / \mathrm{s}$. The reference model bandwidth plays a major role in the compensation quality of the state space predictor. 
A similar study of variations in the damping ratio was completed in a manner similar to varying the bandwidth. This study shows that the damping ratio of the reference model does not make a significant difference in the state space compensation.

The order of the reference model also affects the state space compensation. To demonstrate that, $3^{\text {rd }}$-order and $4^{\text {th }}$-order reference models were compared first. In order to accomplish this, a $3^{\text {rd }}$-order model was formed by reducing a $4^{\text {th }}$-order model in a manner such that the reduced-order model shares similar frequency characteristics with the original model. Then both models were used in the state space compensation. The result shows that the compensation error is considerably greater with the $3^{\text {rd }}$-order model than with its $4^{\text {th }}$-order counterpart. This agrees with the results of the offline simulation tests presented in AIAA 5441(2004) $)^{1}$.

A $2^{\text {nd }}$-order reference model results in even poorer compensation quality than a $3^{\text {rd }}$-order model. With a $2^{\text {nd }}$-order model, the simplified state space filter is given as $y_{p}=a_{0} y+a_{1} \dot{y}+\left(1-\alpha_{0}\right) u$, where $\alpha_{0}$ and $\alpha_{1}$ are solutions of equations $\sum_{i=0}^{1} \alpha_{i} \lambda_{j} t_{d},(j=0,1)$, with $\lambda_{0}$ and $\lambda_{1}$ being the two eigenvalues of the model. The compensation is inferior to that of the state space compensation with a $4^{\text {th }}$-order model (Eq.(5)) because less system information is used for prediction.

Finally, if a $5^{\text {th }}$-oder or higher reference model is employed, the filter state vector contains either a higher frequency jerk component (derivative of acceleration) or triple integration of the aircraft state, which is likely to introduce computational artifacts. Therefore, the $4^{\text {th }}$-order reference model is the best choice for this situation.

The gain of a reference model also influences the compensation quality. From Table 4, the coefficient $c_{3}$ is a function of the gain of the model (i.e., $b_{2}$ ). The large gain of the reference model amplifies the high frequency components in the control input $u$, but the effect is negligible since the $4^{\text {th }}$ term (with coefficient $c_{3}$ ) contributes little to the compensation. In addition, the sensitivity of a reference model's compensation quality to the variation of its gain depends on the individual model: some models are more sensitive than others. In other words, the effect of the gain of a reference model is much less significant than its bandwidth.

In summary, the following points are the conclusions of this study on the state space predictor, and the relations between the compensation quality and the reference model:

1) The state space predictor can be simplified to a general PID filter ${ }^{1}$.

2) The state space compensation may be viewed as a Taylor series extrapolation in the state space form.

3) Satisfactory compensation in simulating the Boeing 757 can be made when the bandwidth is below $1 \mathrm{rad} / \mathrm{s}$. The reference model bandwidth plays a major role in the compensation quality of the state space predictor.

4) The damping ratio of the reference model does not make a significant difference in the state space compensation.

5) The $4^{\text {th }}$-order reference model is the best choice for this situation.

6) The effect of the gain of a reference model is much less significant than its bandwidth.

\section{Transport Delay Measurement}

A series of tests were conducted to measure the transport delays in the Visual Motion Simulator (VMS) at the NASA Langley Research Center, using a device called $\operatorname{SIMES}^{7}$ (the SIMulator Evaluation System). Developed by SAIC for the Naval Air Warfare Center Training Systems Division, in cooperation with the Wright-Patterson Air Force Base, SIMES is a comprehensive, non-intrusive, accurate and reliable system to test, debug, and document the cueing systems and dynamic models of various kinds of simulators. SIMES also can generate control signal in place 
of the operator to run unmanned tests. Thus the SIMES provides a combination of the functions of the control loader, logical analyzer and video level detector. It can generate and send sinusoidal signals and sweep signals to the simulator, and collect responses from it. Unfortunately the SIMES unit is not capable of frequency response analysis (it's not a FRA). Figure 2 shows where the SIMES-generated input was added as well as where various data were collected on the VMS. The control signal was inserted either at point $\mathrm{A}$, so that the aircraft states were input to the cueing systems directly from the SIMES (Scenario I), or at point B, so that the aircraft states were provided by the EOM. In the latter case the aircraft model was either in use (Scenario III) or bypassed by replacing it with a constant gain $\mathrm{K}$ (Scenario II). The SIMES generated input and collected the data (except those EVDAS visual signals) at the speed of $1000 \mathrm{~Hz}$; the mainframe computer update frequency was $40 \mathrm{~Hz}$, and the visual computers ran at a rate of $60 \mathrm{~Hz}$. Therefore, some asynchronous communication delays exist. The EVDAS signals were recorded at $60 \mathrm{~Hz}$.

The numbers in Fig. 2 indicate the points at which the data were collected from the VMS by the SIMES, and they are tabulated in Table 5. Specifically, the five EVDAS signals were obtained in the visual system and were used to measure the visual system transport delay; a photodiode was used to measure the instrument system transport delay; signals 14-19 were used to measure the motion system delay. Points labeled 14-19 are located sequentially in the motion system, as depicted in the lower half of Fig. 2.

The leg positions (labeled 19) were measured with potentiometers, and were then transformed to the position and the orientations of the payload platform using a recursive inverse Newton-Raphson transformation. The transformed position and orientations were then used to determine the delay of the motion system. In addition, six accelerometers mounted on the payload platform sensed accelerations that were transformed to the payload platform accelerations in all six DOF's (labeled 20) using kinematic transformation. The motion system transport delay was also measured from the leg positions sensed by the potentiometers. Because the potentiometer caused larger error than the accelerometer, the final measure result of the motion system delay was from the accelerometer data. A $2^{\text {nd }}$-order filter was applied to the accelerometer data to reduce the measuring noise caused by the accelerometers (labeled 21).

\section{A. Scenario I}

Table 5. The three coefficients calculated with different methods $\left(t_{d}=192 \mathrm{~ms}\right)$

\begin{tabular}{|c|c|}
\hline No. & Name \\
\hline 1 & Control Device Position Over Drive \\
\hline 2 & Control Device Position \\
\hline 3 & Accelerations \\
\hline $4,5,6$ & Rates, Attitudes, Pilot Eye Point \\
\hline $7-11$ & EVDAS Signals \\
\hline 12 & Attitude Indicator \\
\hline 13 & Photo Diode \\
\hline 14,15 & Attitude\& Rate Motion Commands \\
\hline 16 & Leg Drive Commands \\
\hline 17 & Simulator Leg Commands \\
\hline 18 & Accelerometers \\
\hline 19 & Leg Positions \\
\hline 20 & Accelerations from the Accelerometers \\
\hline 21 & Filtered Values of 20 \\
\hline
\end{tabular}

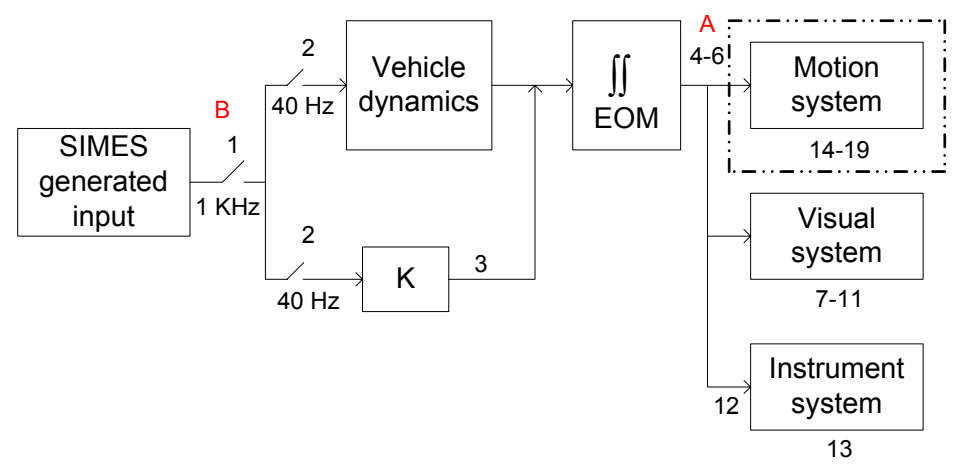

(a)

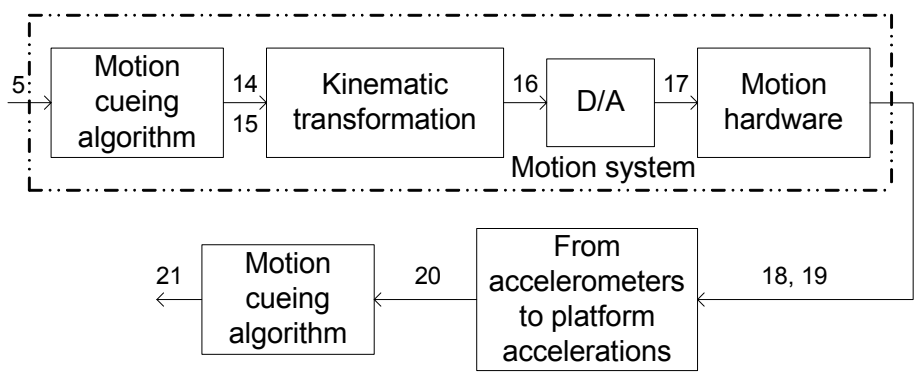

(b)
Fig. 2. Signal flow diagram of the delay measurement in the VMS 
For scenario I, because both the aircraft model and the EOM were skipped, the output signals of the visual system and the instrument system resemble the input. The measurement of the visual delay is based on the EVDAS Y2 signal. The transport delay in the two cue channels were obtained as the average of the delays determined from the 10 transition points, and were further averaged across the five test runs conducted. The result is given in Table 6.

The delay is $58 \mathrm{~ms}$ in the visual channel and $40 \mathrm{~ms}$ in the instrument channel, with a cueing mismatch of $16 \mathrm{~ms}$. In this test, the sampling latencies in the two channels were 14 and $12 \mathrm{~ms}$, both of which are less than one frame $(16.7 \mathrm{~ms})$. They are not counted because the sampling latency behaves as communication asynchrony, which will be addressed later. The theoretical average time delay in the visual is 3.5 frames, that is, $58.4 \mathrm{~ms}$ if the update cycle of the visual system is $16.7 \mathrm{~ms}$. Therefore, the measuring error for the visual delay is small.

The input signal used to measure the delay in the motion system was either a step or a doublet. The terminal responses of the motion system were sensed using accelerometers (for the platform accelerations) and potentiometers (for leg positions). The accelerometer readings were favored due to the large potentiometer latency. Because of the measuring noise in the accelerometers, the data were filtered before determining delays from them. Both the raw and filtered data were employed to determine the delay. The transport delays in the motion system alone with both step input and doublet input are averaged to be $56 \mathrm{~ms}$ (Table 6). Again, a sampling latency of $10 \mathrm{~ms}$ is not included.

\section{B. Scenario II and III}

In both scenarios, the SIMES input signal was injected at point B (Fig. 1), and the difference is whether the aircraft model was skipped (scenario II) or in use. In both cases, the onset of the response was difficult to identify. Therefore, a least squares curve fit was employed to fit different time Table 7. Transport delay in the three basic channels

\begin{tabular}{|c|c|c|}
\hline \multirow{2}{*}{$\begin{array}{c}\text { Aerodynamics } \\
\text { Skipped? }\end{array}$} & Motion & Delay (ms) \\
\cline { 2 - 3 } & 88 & 106 \\
\hline Yes (Scenario II) & 101 & 132 \\
\hline No (Scenario III) & & Visual \\
\hline
\end{tabular}
(he starting point corresponding to the onset of the input. Doublet signals with varied duration were used as input signals. The total transport delays from the aircraft control input to the motion terminal and visual terminal, including the sampling uncertainty and the asynchrony, are given in Table 7. All dynamic model processes, EOM integrations and various coordinate transformations are completed in a single frame, and the delay of most intermediate variables can be determined with small errors. Processing of the aerodynamic model only consumes $7 \mathrm{~ms}$ of a frame.

\section{Summary}

Notice that the values in the Table 7 are averages with large variances due to the difficulty of determining the onset of the response and the measurement noise. It suggests that measuring the total transport delay as was done in scenarios II and III is not a good solution. This assertion is confirmed by the fact that the frame length of the simulation mainframe computer may be altered occasionally, depending on the aircraft model and simulation task (More complex aircraft models may require longer a frame time). This means that the total transport delay measured in one situation does not necessarily apply to another. Fortunately, this problem may be solved given that: 1) the visual system computer's frame is fixed to $16.7 \mathrm{~ms}$, and the delays in the individual cueing channels can be measured accurately; 2) the delays associated with the aircraft states from the aerodynamics and the EOM can be determined with high accuracies. Therefore, a good measure of the total transport delay can be obtained using two steps: first, measure the individual cueing subsystem delay as in scenario I, and second, calculate the total delay with

$$
t_{d}=1.5 T_{m}+\bar{t}_{a}+t_{c}
$$

where $T_{m}$ is the frame cycle of the mainframe computer, $\bar{t}_{a}$ the average asynchronous delay, and $t_{c}$ the transport delay in a cueing channel. On the average it takes half a frame for a control input change to be sensed by the 
mainframe computer (this is known as sampling latency), and one frame to complete the aerodynamics and the EOM, so that the total average delay in updating the aircraft states is $1.5 T_{m}, \bar{t}_{a}$ may be calculated with Eq. (18).

$$
\bar{t}_{a}(m)=T\left\{m \frac{M}{N}-\left\lfloor m \frac{M}{N}\right\rfloor\right\}
$$

where $m$ is the iteration index of the visual computer, $T$ is the update period of the host computer, and $M / N$ is the minimal fraction equal to the ratio of $T^{\prime} / T$ ( $T^{\prime}$ is the update period of the visual computer), or $M$ and $N$ must be relatively prime ${ }^{8}$. For this measurement, $T_{m}$ is $25 \mathrm{~ms}$, and the for the final piloted tests, it was set to $16 \mathrm{~ms}$. The values of all three terms in Eq. (17), the maximums and minimums, if any, and the total delays in the three basic cueing channels for these two mainframe computer frames are summarized in Table 8.

Therefore, the average total delay (baseline delay) in the visual channel in the final tests will be $90 \mathrm{~ms}$ (approximation of $89.7 \mathrm{~ms}$ highlighted in red).

Table 8. Time delays of different sources and the total delays

\begin{tabular}{|c|c|c|c|c|c|c|c|c|c|c|c|}
\hline \multirow{3}{*}{$\begin{array}{c}\text { Main } \\
\text { frame } \\
(\mathrm{ms})\end{array}$} & \multirow{3}{*}{ Cue } & \multicolumn{10}{|c|}{ Delay (ms) } \\
\hline & & \multicolumn{3}{|c|}{$t_{m}$} & \multicolumn{3}{|c|}{$t_{a}$} & \multirow{2}{*}{$t_{c}$} & \multicolumn{3}{|c|}{ Total $t_{d}$} \\
\hline & & Max & Ave. & Min & Max & Ave & Min & & Max & Ave. & Min \\
\hline \multirow{3}{*}{25} & Visual & \multirow{3}{*}{50} & \multirow{3}{*}{37.5} & \multirow{3}{*}{25} & \multirow{3}{*}{16.7} & \multirow{3}{*}{8.3} & \multirow{3}{*}{0} & 58 & 124.7 & 103.8 & 93 \\
\hline & Motion & & & & & & & 56 & 122.7 & 101.8 & 91 \\
\hline & Instrument & & & & & & & 40 & 106.7 & 85.8 & 75 \\
\hline \multirow{3}{*}{16} & Visual & \multirow{3}{*}{32} & \multirow{3}{*}{24} & \multirow{3}{*}{16} & \multirow{3}{*}{15.3} & \multirow{3}{*}{7.7} & \multirow{3}{*}{0} & 58 & 105.3 & 89.7 & 84 \\
\hline & Motion & & & & & & & 56 & 103.3 & 87.7 & 82 \\
\hline & Instrument & & & & & & & 40 & 87.3 & 71.7 & 66 \\
\hline
\end{tabular}

\section{Results of Final Piloted Tests}

The purpose of the piloted tests was to assess the effectiveness of the two novel predictors along with the McFarland compensator, and to compare the results with those of the offline tests that were introduced on AIAA $5441(2004)^{1}$. In addition to the above three predictors, an expedient algorithm was applied to the McFarland predictor in an attempt to reduce the spikes which result from high frequency input. Thus, four distinct compensators were tested: McFarland predictor, spike-reduced McFarland predictor, adaptive predictor, and state space predictor. For the remainder of this paper, they may be called MF, MFR, AP and SS, respectively.

\section{A. Test Procedure}

Thirteen subjects with varying aircraft and flight experience flew the NASA Langley Research Center Visual Motion Simulator (VMS) running a high fidelity, highly nonlinear mathematical model of a Boeing 757-200. The pilots consisted of three NASA simulator engineers, three NASA research pilots, two corporate pilots, one FAA pilot, and four commercial pilots. The schedule for each pilot was: on the first day, three hours for training; on the second day, six hours for experiment.

Each pilot executed two flight scenarios - a straight-in approach and an offset approach. Wind was added to the straight-in approach, rotating 180 degrees from head on to tail. Turbulence and a gust at the threshold were added to the offset approach. Artificial delays of 0, 48, 96 and $192 \mathrm{~ms}$, all integer multiples of the update period (16 ms), were inserted into the visual channel in addition to the $90 \mathrm{~ms}$ baseline delay, resulting in total delays of $90,138,186$ and $282 \mathrm{~ms}$. The added delay will be referred to as "short" delay when it is 0 or $48 \mathrm{~ms}$, or "long" delay when it is 96 or 192 ms. There were five compensation options: no compensation (NC), MF, MFR, AP and SS. Therefore, in each maneuver, there were 20 test runs [4 (delays) by 5 (compensation algorithms)]. Therefore, each pilot ran 40 tests in 
total. For convenience of statement, the four added delay cases from 0 to $192 \mathrm{~ms}$ will be referred to as case I to case IV for the straight-in approach, and case V to VIII for the offset approach.

Four types of analyses of the collected data were completed in order to evaluate the results. They are: 1) the pilots' performance in minimizing the glide slope error (GSE) and touchdown point error (TDE); 2) the pilots' handling qualities rating from the Cooper-Harper Ratings (CHR) on the glide slope and touchdown; 3) the pilots' workload from the NASA Task Load Index (TLX); and 4) the pilots' control workload based on the power spectral density (PSD) of the simulator control inputs. The first and last assessments are objective evaluations and the midtwo are quasi-objective evaluations. In all these metrics, larger value implies poorer simulation performance. The succeeding sub-sections treat the analyses and the results of the final tests in this order. The analyses and comparisons fall into two categories: 1) compare each compensation case with the no-compensation case; 2) compare the MFR, AP and SS with the MF.

\section{B. Analyses of the Glide Slope Error (GSE)}

In both approach maneuvers, the aircraft, starting from an initial altitude of $1303 \mathrm{ft}$, descends gradually to approach the designated runway for landing. The angle that the aircraft deviates from the ideal glide slope defines the glide slope error. The integrated PSD and root mean squared error (RMSE) of the GSE were calculated, and because the PSD pf the GSE shows more distinction among cases, the analysis is based on the PSD of the GSE. The integrated PSD is the integration of the PSD over the frequency range in which it is distributed (it usually is from 0 to $1 \mathrm{~Hz}$ ).

Because the 13 pilots revealed large variations in the GSE due to the widely differing control strategies employed, the GSE data from five pilots, i.e., pilots \# 1, 2, 5,11 and 12 were selected since they resulted in more positive compensation in terms of the GSE. Figure 3 shows the mean values and standard deviations (STD) of the glide slope error of the selected pilots with different compensation algorithms. Compared with the no-compensation case, the McFarland predictor revealed decreased mean GSE for six cases (case I, III, IV, V, VII \& VIII), the MFR revealed decreased mean GSE for five cases (case III, IV, V, VI $\&$ VII), the adaptive predictor revealed

Mean \& STD of Glide Slope Error (in PSD) for Selected Pilots (\# 1,2,5,11,12): Straight-in

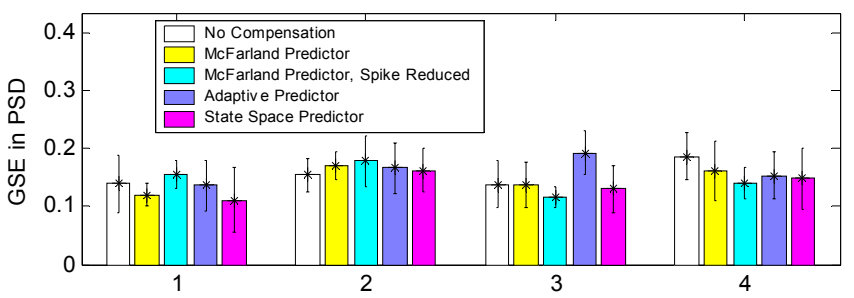

Offset Approach

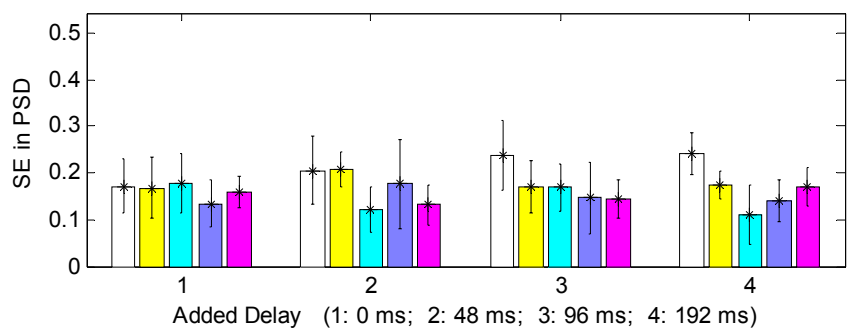

Fig. 3. Mean \& STD of the glide slope error for pilots \#1, 2, 5, 11 \& 12

decreased mean GSE for six cases (case I, IV, V, VI, VII \& VIII), and the SS revealed decreased mean GSE for seven cases (case I, III, IV, V, VI, VII \& VIII). Significant compensation is found for the MFR and AP for case VIII. All compensators resulted in better glide slope performance in terms of mean GSE. Compared with the MF, the MFR showed lower mean GSE for five cases (case III, IV, VI, VII \& VIII), the AP showed lower mean GSE for six cases (case II, IV, V, VI, VII \& VIII), and the SS showed lower mean GSE for all eight cases. The MFR slightly improved McFarland compensation results. Improved effectiveness with the two new novel predictors is apparent.

\section{Analyses of the Touchdown Error (TDE)}

As the aircraft touches down, the difference between the actual aircraft $x$ and $y$ position and the ideal $x$ and $y$ position is defined as the touchdown errors. The subjects were instructed to touchdown within the "touchdown box" of $1000 \times 140 \mathrm{ft}^{2}$. The $x$ touchdown error is defined as the absolute value of the difference between the $x$ coordinates of the touchdown point and the center of the box. Because the pilots were instructed to pay more attention to the $x$ TDE, only the $\mathrm{x}$ TDE is illustrated here. For the remainder of this paper, the TDE only indicates the $x$ TDE. The TDE results also were from all 13 pilots.

Figure 4 shows the mean values and standard deviations of the $x$ touchdown error of the selected pilots with different compensation algorithms. Compared with the no-compensation case, the McFarland predictor revealed decreased mean TDE for two cases (case IV \& VIII), the MFR revealed decreased mean TDE for four cases (case I, 
VI, VII \& VIII), the adaptive predictor revealed decreased mean TDE for six cases (case I, III, IV, VI, VII \& VIII), and the SS revealed decreased mean TDE for five cases (case I, III, VI, VII \& VIII). The MF resulted in poor compensation results, because it increased the mean TDE for six cases. The two novel predictors resulted in good compensation. Compared with the MF, the MFR showed lower mean TDE for five cases (case I, II, III, VI \& VII), the AP showed lower mean TDE for seven cases (case I - VII), and the SS showed lower mean TDE for seven cases (case I, II, III, V, VI, VII \& VIII). The MFR slightly improved the McFarland compensation. Improved effectiveness with the two new novel predictors is apparent.

\section{Analyses of the Cooper-Harper Rating (CHR)}

Two sets of CHR were collected in the final piloted tests: the CHR of maintaining the glide slope and the CHR of manipulating the touchdown point. The difference between the two Cooper-Harper ratings reveals no considerable difference. Therefore, only the
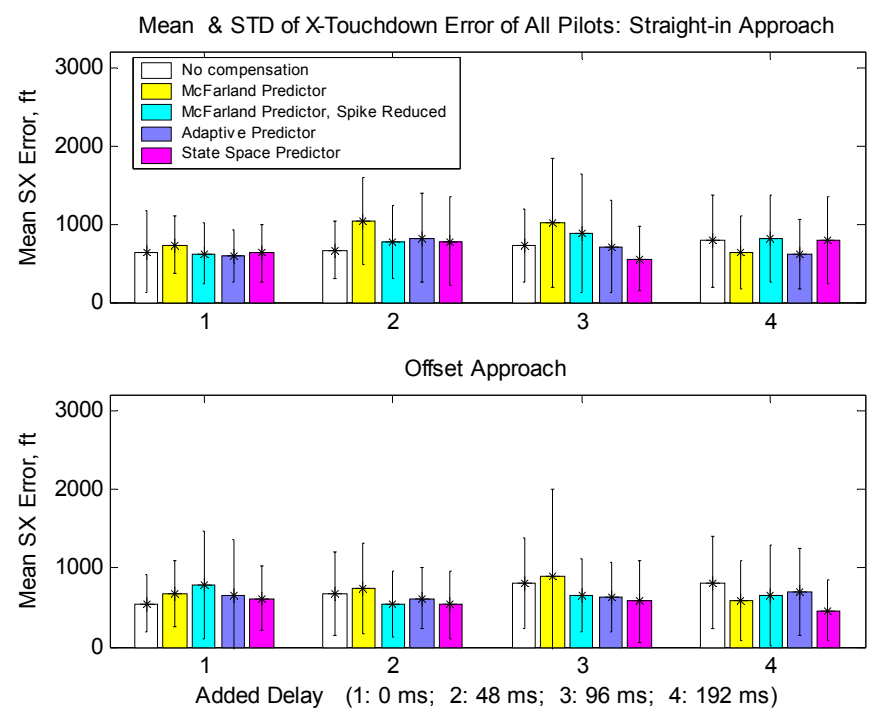

CHR on the touchdown is illustrated here. Figure 5 shows the mean values and standard deviations of CHR on the touchdown for different compensations in the straight-in approach. As a comparison, the corresponding TDE is shown in the lower half of this figure. Compared with the no-compensation case, the McFarland predictor revealed decreased mean CHR for six cases (case I, II, III, IV, VI \& VII), the MFR revealed decreased mean CHR for five cases (case III, IV, VI, VII \& VIII), the adaptive predictor revealed decreased mean CHR for four cases (case III, IV, VII \& VIII), and the SS revealed decreased mean CHR for five cases (case I, III, VI, VII \& VIII). There were several cases for which some predictors showed equal mean CHR as the no-compensation case: they are case VIII for the MF, case VI for the AP, and cases II $\& \mathrm{~V}$ for the SS. Compared with the MF, the MFR showed lower mean CHR for three cases (case III, VII \& VIII), the AP showed lower mean CHR for three cases (case IV, VII \& VIII), and the SS showed lower mean CHR for six cases (case III - VIII). In terms of the CHR, the MF was slightly better in compensation than the MFR and AP. Improved effectiveness with the SS is fairly apparent. Comparing the results of CHR with those of TDE and GSE shows that the objective performance metrics showed more obvious improvement of the two novel predictors.

\section{E. Analyses of the NASA TLX}

The NASA Task Load Index (Hart and Staveland in $1988^{\circ}$ ) is a multi-dimensional quasi-objective metric to rate the overall workload of the operator in a man-machine interaction. The TLX is a weighted average of six ratings of six subscales of
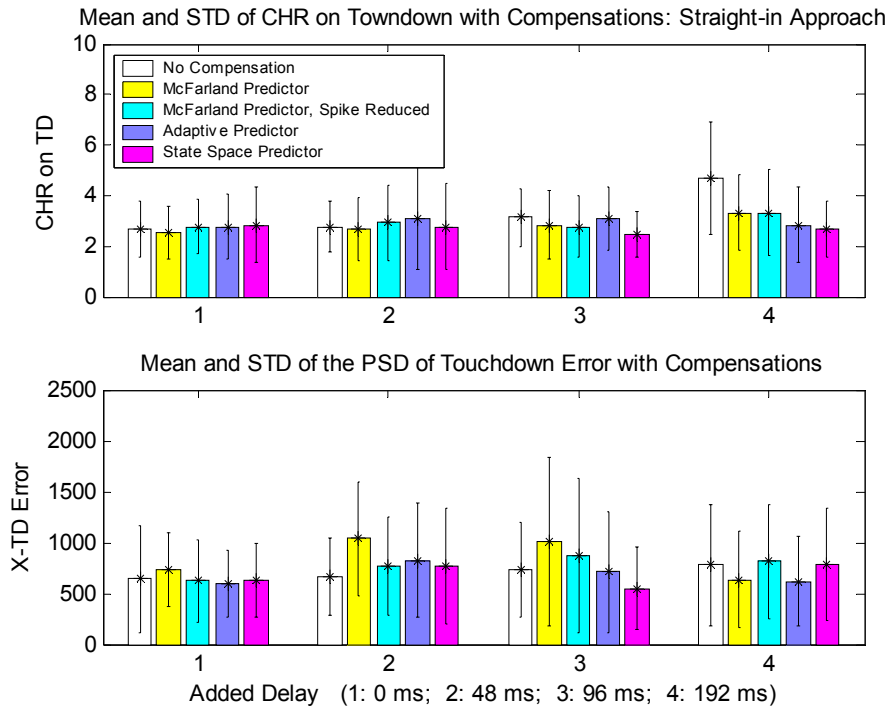

Fig. 5. Mean \& STD of CHR \& TDE for 13 pilots: straight-in approach

workload $^{10}$ : mental demand, physical demand, temporal demand, performance, effort and frustration. High TLX indicates high pilot's workload. 
Figure 6 shows the difference in the mean value and standard deviation of the TLX when using the four types of compensators. Compared with the no-compensation case, the McFarland predictor revealed decreased mean TLX for four cases (case III, IV V \& VII), the MFR revealed decreased mean TLX for six cases (case I, II, III, IV, VII \& VIII), the adaptive predictor revealed decreased mean TLX for five cases (case III, IV, V, VII \& VIII), and the SS revealed decreased mean TLX for five cases (case II, III, IV, VI \& VIII). Compared with the MF, the MFR showed lower mean TLX for five cases (case I, II, III, VII \& VIII), the AP showed lower mean TLX for five cases (case IV VIII), and the SS showed lower mean TLX for five cases (case I, II, III, VI \& VIII). The MFR, AP and SS showed fairly improved the compensation compared with MF. The results from the TLX analyses were similar to those from the other quasi-objective metric, the CHR.

\section{F. Analysis on PSD of Pilot Controls}

Smoothed periodogram algorithms were used to evaluate the PSD of the pilot controls: the roll and pitch sticks, the rudder pedal and the throttle. Integrated PSD were calculated over the interval from 0 to $1 \mathrm{~Hz}$ because the power outside this range was small for all four controls. The PSD of the throttle showed little obvious and consistent changes with the amount of delay and the compensation. Considering the paper length, only the integrated roll stick PSD is illustrated here.

Figure 7 shows how the compensators affect the mean values and standard deviations of the integrated PSD of the roll stick. In the following statements in this subsection (F), PSD implies PSD of the roll stick. Compared with the no-compensation case, the McFarland predictor revealed decreased mean PSD for five cases (case III, IV, V, VII \& VIII), the MFR revealed decreased mean PSD for six cases (case II, III, IV, V, VII \& VIII), the adaptive predictor revealed decreased mean CHR for six cases (case II, III, IV, V, VII \& VIII), and the SS revealed decreased mean PSD for six cases (case III VIII). Compared with the MF, the MFR showed lower mean PSD for four cases (case I, II, IV \& VI), the AP showed lower mean CHR for five cases (case I, II, IV, VI \& VIII), and the SS showed lower mean CHR for six cases (case II, III, IV, V, VI,
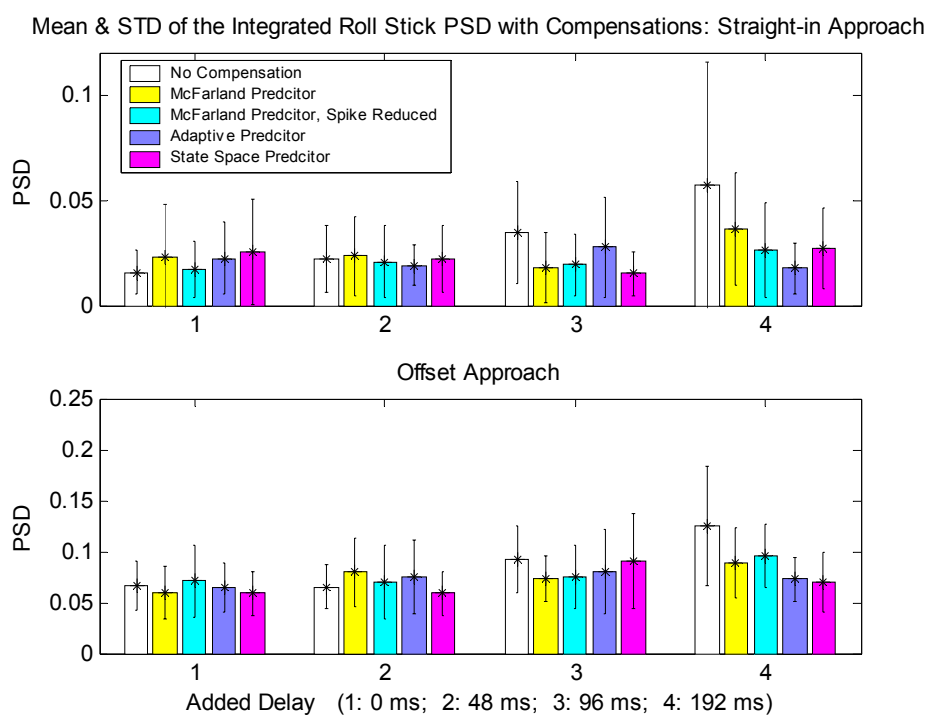

Fig. 7. Mean \& STD of the integrated roll stick PSD for all 13 Pilots

VIII). In terms of the PSD, the MFR produced inconsistent comparison results compared to the MF. Improved effectiveness over the MF is considerably obvious with the SS, and is fairly obvious with the AP.

\section{G. Summary of Piloted Tests Results}

For compactness, not all results of the piloted tests are illustrated in this section. Therefore, a summary of the whole piloted tests analyses is presented as follows. The summary is based on analyses with respect to ten sub- 
metrics: GSE, TDE (in X direction), CHR on GS (CHRGS), CHR on TD (CHRTD), TLX, integrated PSD of the roll stick (IPSDRS), pitch stick (IPSDPS) and rudder pedal (IPSDRP), and the frequencies of the highest PSD peak of the roll stick (FPSDRS) and pitch stick (FPSDPS).

1) In general, inserting artificial time delay increased mean measures in all sub-metrics. However, the increase is not obvious for short delay cases in terms of most submetrics (Figure 8 shows one example).

2) The time delay tends to move the highest PSD peak to higher frequency, and longer delay produced larger movement, whereas the compensation moves the highest PSD peak back to lower frequency, and the movement is slightly more obvious with the two novel predictors than with the MF. These results agree with the preliminary tests ${ }^{11}$.

3) On average, the delay increases the integrated PSD in the frequency interval from 0.022 to $0.614 \mathrm{~Hz}$ for the straight-in approach, and the interval is from 0.08 to $0.528 \mathrm{~Hz}$
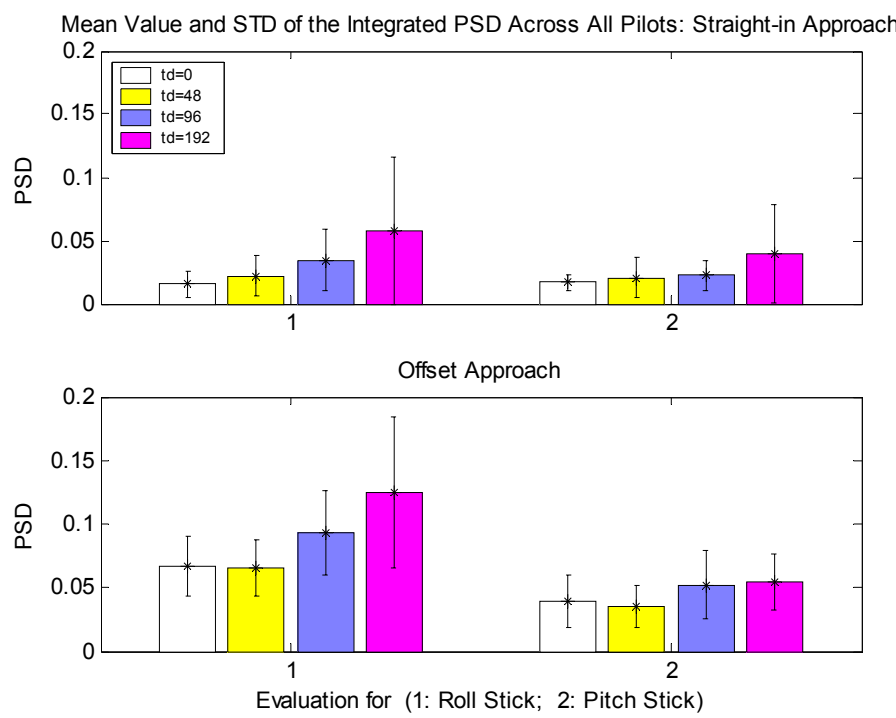

Fig. 8. Mean value \& STD of the integrated PSD changing with the time delay for the offset approach. On the other hand, the compensation decreases the integrated PSD in the interval from 0.068 to $0.559 \mathrm{~Hz}$ for the straight-in approach, and from 0.078 to $0.524 \mathrm{~Hz}$ for the offset approach. The effective intervals for the offset approach are narrower than those of the straight-in approach. In addition, the SS showed wider effective intervals than all other compensators. Both novel predictors showed slightly wider intervals than that of the MF. This is further evidence that the two novel predictors can achieve better compensation results than the McFarland predictor.

4) The overall analyses based on all ten sub-metrics show that while the adaptive predictor results in slightly poorer compensation for short added delay and better compensation for long added delay than the McFarland compensator, the state space predictor is fairly superior for "short" delays and significantly superior for "long" delays to the McFarland compensator. The state space predictor also achieves better compensation than the adaptive predictor. The results of the evaluation on the effectiveness of these predictors in the piloted tests agree with those in the theoretical offline tests conducted with the recorded simulation aircraft states.

5) Large variation in the STD in almost all sub-metrics might result either from different control strategies employed by different pilot subjects, or from the metrics being inconclusive. Figure 8 shows larger variation with the effect of the time delay. It indicates that for some pilots, the performance was even better with 192 ms added delay than without added delay. This demonstrates that the metrics used are not sufficiently effective. The variations with compensation are expected to be greater than those with the time delay, because no compensation can restore the system performance one hundred percent.

\section{Conclusion}

The paper demonstrated mathematically why the stochastic approximation algorithm achieves the best compensation results among all the adaptive predictors using the asymptotic ODE of the stochastic approximation algorithm. The relationship between the reference model and the state space compensator performance was investigated in great detail, showing that the bandwidth of the reference model plays a key role in deciding the compensation quality of the state space predictor. The baseline visual image delay was measured to be $90 \mathrm{~ms}$.

Piloted simulation tests were conducted for assessing the effectiveness of the two novel compensators in comparison to the McFarland predictor and no compensation. Thirteen pilots with heterogeneous flight experience executed straight-in and offset approaches, at various delay configurations, on a flight simulator where different 
predictors were applied to compensate for transport delay. Four metrics - the glide slope and touchdown errors, power spectral density of the pilot control inputs, NASA Task Load Index, and Cooper-Harper rating on the handling qualities were employed for the analyses. The overall analyses show that while the adaptive predictor results in slightly poorer compensation for short added delay and better compensation for long added delay than the McFarland compensator, the state space predictor is fairly superior for short delay and significantly superior for long delay to the McFarland compensator. The state space predictor also achieves better compensation than the adaptive predictor. The results of the evaluation on the effectiveness of these predictors in the piloted tests agree with those in the theoretical offline tests conducted with the recorded simulation aircraft states.

\section{References}

\footnotetext{
${ }^{1}$ Guo, L., Cardullo, F. M., Houck, J., A., Kelly, L., C., Wolters, T. "New Predictive Filters for Compensating the Transport Delay on a Flight Simulator", AIAA-5441, 2004

${ }^{2}$ McFarland R.E., Transport Delay Compensation for Computer-Generated Imagery Systems, NASA Ames Research Center, 1988

${ }^{3}$ Sobiski, D., J., and Cardullo, F., M., "Predictive Compensation of Visual System Time Delays", Proceedings of the AIAA Flight Simulation Technologies Conference, Washington, DC, 1987

${ }^{4}$ Sobiski, D., J., Master Thesis, State University of New York at Binghamton, Binghamton, NY, 1988

${ }^{5}$ Sinha, N., K., and Kuszta, B., "Modeling and Identification of Dynamic Systems", Van Nostrand Reinhold Company, 1983

${ }^{6}$ Kushner, G. and George Yin, "Stochastic Approximation Algorithms and Applications", New York, Springer, 1997

${ }^{7}$ Wolters, T. E, "Research Flight Deck Transport Delay Study", Simulation Systems Branch, Simulation and Research Aircraft division

${ }^{8}$ McFarland R., E., CGI Delay Compensation, NASA Ames Research Center, 1986

${ }^{9}$ Hart, S. G., and Staveland, L. E. (1988). Development of a Multi-dimensional Workload Rating Scale: Results of Empirical and Theoretical Research. In P. A. Hancock, and Meshkati, N. (Ed.), Human Mental Workload. Amsterdam, The Netherlands: Elsevier.

${ }^{10}$ Middendorf, M., M., Fiorita, A., L., and McMillan G., R., “The Effects of Simulator Transport Delay on Performance, Workload, and Control Activity", AIAA-91-2965, 1991

${ }^{11}$ Guo, L., Cardullo, Telban, J., R., Houck, J., A., Kelly, L., C., "The Results of a Simulator Study to Determine the Effects on Pilot Performance of Two Different Motion Cueing Algorithms and Various Delays, Compensated and Uncompensated", AIAA-5676, 2003
} 